\title{
Simple Complexities: A Rule-based Approach to Architectural Design
}

\author{
Marco Hemmerling \\ Hochschule Ostwestfalen-Lippe Univ. of Applied Sciences \\ marco.hemmerling@hs-owl.de
}

\begin{abstract}
The paper discusses rule-based design strategies that allow for the generation of spatial complexity based on simple principles and taking as well the parameters of construction into account. The presented case studies as part of the academic project "Simple Complexities" focused on the early integration of optimization parameters, regarding structural performance, physical properties and material specification as well as aspects of fabrication to inform the architectural design. The clear conception of a computation-process whose rules lead to certain formal and structural consequences is the necessary first step towards an architecture that is both structurally interesting and systematically coherent.
\end{abstract}

Keywords: Performance based design; Rule-based design; Computational Design and construction; Complexity.

\section{Introduction}

Since nearly three decades the broad application of computational design and construction technology has widely changed the use and perception of computer software in architecture. Thus, we have seen a shift from mere drawing-tools towards mighty parametric design methods. These tools have allowed many architects for the conception and design of very complex architectural projects. And so, many formally intricate buildings have been designed and built over the past years. And many of these projects have undergone massive so-called postrationalization-processes - i.e. methods of (mostly) intelligent geometrical simplification, like the costly triangulation of doublecurved surfaces. From today's point of view such processes, however extremely elaborate in themselves, appear to be a bit anachronistic. They seem like attempts of after-computerization of actually post-modernist design approaches. However, Patrik Schumacher describes the validity of parametric design as an important movement within the history of architecture, when he states: "Contemporary avant-garde architecture is addressing the demand for an increased level of articulated complexity by means of retooling its methods on the basis of parametric design systems" (Schumacher, 2010). Indeed, much of the attention in the architectural discourse of recent years has been relegated to the field of aesthetics and high levels of interest in the generation and articulation of complex geometric configurations. Nevertheless, beyond geometry, complexity involves much more invisible layers of interaction as well as interdependencies and non-linear responses. Furthermore, complexity in architecture is often confused with complicated structures. In fact complexity does not require complicated systems and intricate rules to unfold its potential. On the contrary complications lead to multiplicative chains of unanticipated effects rather than generating a practical and robust system. In other words, redundancy and overcompensation - as characteristics of a so-called perfomative system - can solitary be achieved by focusing on the relational qualities and traceable interdependencies, thus the conditions of a system.

\section{Simple Complexities}

Neither the discourse on complexity in architecture is new, nor the discussion about the reference system of architectural design. The Post-Modern movement approached complexity by emphasizing the importance of the context for architecture (Venturi, 1966) and by contrasting it with the universal Modern science of simplicity and reduction (Jencks, 1997). At about the same time the advent of the trans-disciplinary movements of cybernetics and structuralism introduced the idea of regulatory systems to the field of architecture, which since the 1990s experience a revival, as the available computational means and methods enable a more comprehensive and integral approach towards rule-based design and complexity (Valena, 2011). Whereas the Structuralism of the 1970 s encountered limits in complexity that were insurmountable at the time, today there is much to suggest that the return to this apparently unfinished project is causally connected to information technology, which has opened up new possibilities for dealing with complexity.

Managing complexity, negotiating multiple aspects of a design problem and generating various solutions through computational processes enable the architect today to develop a relational and adaptable architecture of interdependencies. Bernard Tschumi's statement "Architecture is not about conditions of design, but about the design of conditions" (Tschumi, 1995) can be seen as a programmatic testimonial for this reference system of architectural design. Against this background the academic project "Simple Complexities" focused on the early integration of optimization parameters, regarding structural performance, 
physical properties and material specification as well as aspects of fabrication to inform the architectural design. The clear conception of a computation-process whose rules lead to certain formal and structural consequences is the necessary first step towards an architecture that is both structurally interesting and systematically coherent. Thus, in this seminar the students attempted to develop strong and fresh architectural projects from fairly simple methods of parametrical definition of space-defining surface-geometry. And while doing so a conceptual, historical, theoretical, and technical framework was built around the projects to take each design beyond the mere development and application of computer-tools.

However, recent software packages (such as Rhinoceros/ Grasshopper) allow for the designing of all kinds of shapes it appears that great spatial or structural effects can be achieved with much simpler geometrical operations if applied creatively. Therefore the project focused on research-based design-strategies that aim to unfold hidden complexities of (at-first-sight) rather simple surface definitions. In this case the formation, variation and intersection of developable and/or ruled surfaces. In architectural history we find a great amount of examples for the use of these surfaces: be it Gaudi and his vaults for the Sagrada Familia in Barcelona, be it Eladio Dieste's Brickwalls, Shukhov's hyperbolical tower, or recent examples, like matterstudio's "La Voûte de LeFevre" at Knowlton University. All examples show a great degree of spatial effects and apparent formal complexity while the architecture's geometry is in fact comparably simple.

\section{Case Studies}

Starting from a surface-based design strategy for a sheltering structure by only using developable and/or ruled surfaces in different combinations/variations/intersections lead to a reinterpretation an generation of complex geometries, based on traceable and at the same time adaptable geometries. With this the aim was to create formal/structural transitions to form and to construct diversified spaces. The starting point for the project was a case-study-research of an existing or conceived building/structure (such as the above...) to gain in-depth understanding of the main geometrical principles that structure the building. The next steps involved the translation of the analysis and its found geometrical principles into new computational design strategies and the development of generic tools for formfinding and structural definition. Aspects of research involved the surface construction as well as the interconnection of different surfaces. Another field of investigation was related to the material of the surfaces. In how far might material-specific parameters (such as residual stress in plywood or metal) help to reinform/alter the design strategy?

The tectonic strategy was enhanced by the use of various plug-ins to the 3D-modeling software Rhinoceros/ Grasshopper (e.g. Karamba, Galapagos, Hoopsnake) as well as software to analyze external parameters like sun radiation and wind load (e.g.
Ecotect). In addition programming tools (e.g. Python) were used to optimize and simplify operations and generate an automatized process for the production of building parts (e.g. numbering and nesting of elements). As a result of this computational design process the students developed very different concepts - both in the architectural/geometrical expression as well as in the structural performance and materiality. However, all of them are robust in the interdependencies between geometry, material and structural performance. And at the same time flexible in the way the structure reacts to external and internal parameters (e.g. adaption to different use, formal changes, reaction to different light ambiences).

\section{Structural enhancement}

The analysis of the intersecting hyper-parabaloids of Felix Candela's restaurant at Xochimilco in Mexico was the starting point for a structural optimization and design process in Manuel Sotomayor's project. The original structure is formed from the saddle shape of eight point-symmetric "hypars". The term hypar, introduced by Heino Engel in his book Structural Systems (Engel, 1967), to mean a hyperbolic paraboloid shape, or more formally a partial hyperbolic paraboloid, cut from the full infinite surface. These seemingly complex shapes can all be constructed from straight lines - and in Candela's case with a resulting concrete shell as thin as just forty millimeters along the visible edges. The geometric principle supports not only the construction and assembly of the structure, but also the spatial continuity of the building. However, the structural performance of the shell is based on a differentiation between the overarching shell and the intersection lines where the loads congregate. Based on the analysis of the structure a design strategy was developed that focused on the equal distribution of stresses throughout the whole shell. Therefore the intersection was dissolved using inverse hyperbolic paraboloids as transition surfaces, referring to Gaudi's design for the Colònia Güell near Barcelona (Moussavi, 2009).
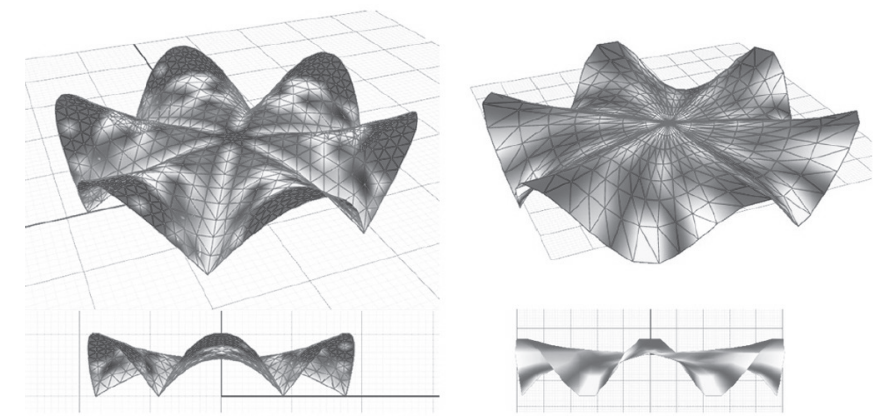

Figure 1: Stress diagram of Candela's structure (left). Structural model with consistent distribution of loads over the shell through interconnected hyperbolic paraboloids (right).

Next to the geometric advancement for the connection of the shells the research focused on a structural optimization by a physics driven design. Thus, the given geometric principle of ruled surfaces was compared to a tensile structure developed by using 
finite element calculations and optimization algorithms in Karamba and Dlubal R-FEM. The geometry of the resulting shape differed just slightly from the original hypars, but the structurally performance of the shell increased significantly.
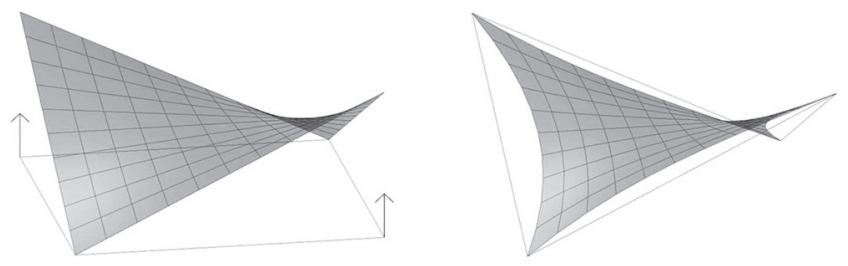

Figure 2: Ruled surface (left) vs. tensile structure (right).

Based on this form-finding principle a set of tensile structures was developed to generate a seemingly complex spatial configuration, which consists of intertwining shell structures, supported by linear compression members. Since the performance of the each membrane is depending on the distribution of stresses throughout the overall structure the further optimization process was carried out using physical form-finding engines taking material parameters into account.

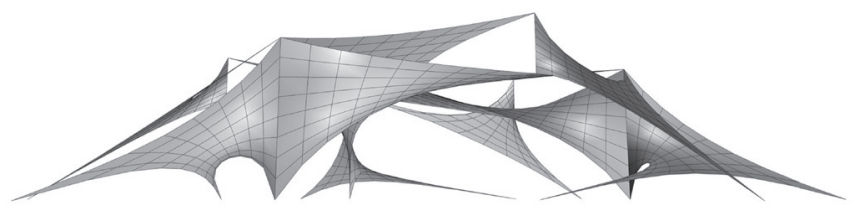

Figure 3: Structurally optimized membrane system.

The first physical engine creates a spring system that finds an equilibrium shape for each membrane according to the given stiffness values. The resulting geometry and the values are sent to a Finite Element Analysis (FEA), which assess the stresses each membrane transfers to its neighbors and the stability of the floating masts (linear compression members). Finally a genetic algorithm is implemented that, through iterations, generates an ideal set of stiffness values for the membranes. The computational approach has been tested and proved in a physical scale model so far and will be developed further in a first 1:1 prototype.

\section{Rule(-base)d surfaces}

The Taichung metropolitan opera house, designed by Japanese architect Toyo Ito, served as a case study for the design research project by Patric Günther. The geometric complexity of the building resulted in a series of complication throughout the realization process, involving an explosion of costs and major delays in the time schedule. The proposed construction method for the curved wall-structure is based on a main steel frame with expanded metal meshes that act as a faceted formwork for a shotcrete layer (sprayed concrete of $250-350 \mathrm{~mm}$ ). The given construction technique is not only time-consuming and needs a lot of rework to achieve the desired result, moreover it lacks a clear strategy that takes into account both - the geometric complexity as well as the circumstances of realization in an all-embracing approach. Therefore the further research focused on the underlying geometric principles that generate the double-curved structure in order to develop a more efficient and at the same time spatially intriguing design strategy.

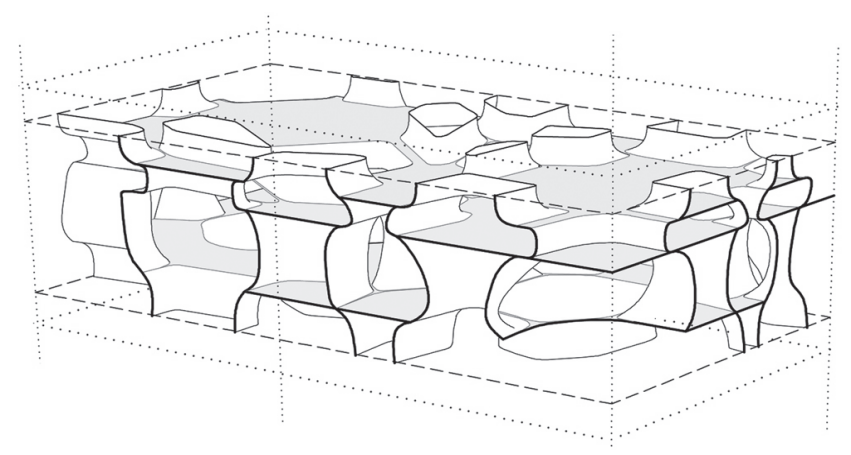

Figure 4: Design model of the Taichung opera house by Toyo Ito.

The underlying geometric principle of the building is based on the Voronoi tessellation (Voronoi, 1908). A horizontal cell-grid is stacked and shifted (e.g. A-B-A to B-A-B) from floor to floor to generate a three-dimensional space-grid. The 3D-Modell constitutes by connecting the polygonal outlines (e.g. A-A and B-B) with twisted surfaces. A series of studies was carried out to investigate modeling techniques, based on ruled surfaces, that allow for a similar spatial result, while offering a fairly simple construction method. Alternating between complex and simple shapes different geometries where examined, due to their spatial and structural properties. The geometry of a catenoid (a minimal surface of rotation, which is defined from two points in a half plane, whose boundary is the axis of revolution of the surface) is quite similar to the proposed geometry for the opera house and at the same time mathematically defined in the Euclidean space. However, it is not a ruled surface that can be undeveloped or constructed from straight elements. On the contrary, the hyperboloid and the hyperbolic paraboloid are doubly ruled surfaces that allow for an efficient construction as described above and achieve a similar, yet more differentiated spatial effect. Based on these findings a computational design strategy was carried out that allowed for the generation of doubly curved surfaces.
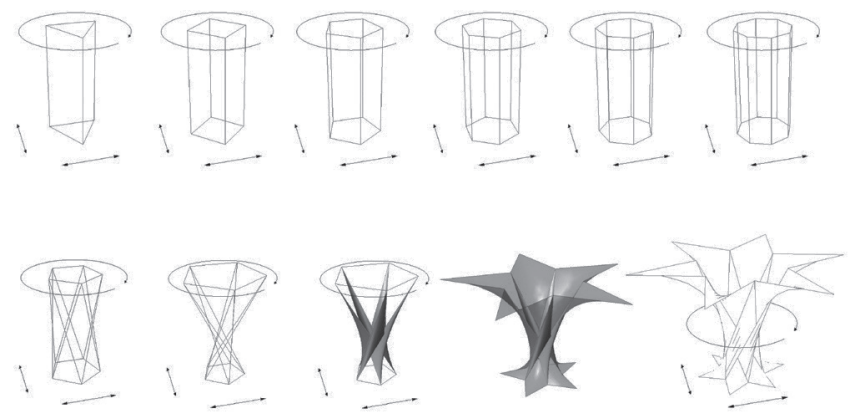

Figure 5: Design evolution of the structural model, built from hyperbolic paraboloids. 
As a result a spatial model was programmed, consisting of connected HP-surfaces that change their orientation from inside out while developing from one floor to the next. In a further process the structural performance of the system was analyzed and optimized (Karamba) and different material parameters (such as wood/steel in different dimensions) were examined in order to minimize the mass and the displacement for the construction. In a first physical scale model the principle was applied to a roof structure, made from linear elements.

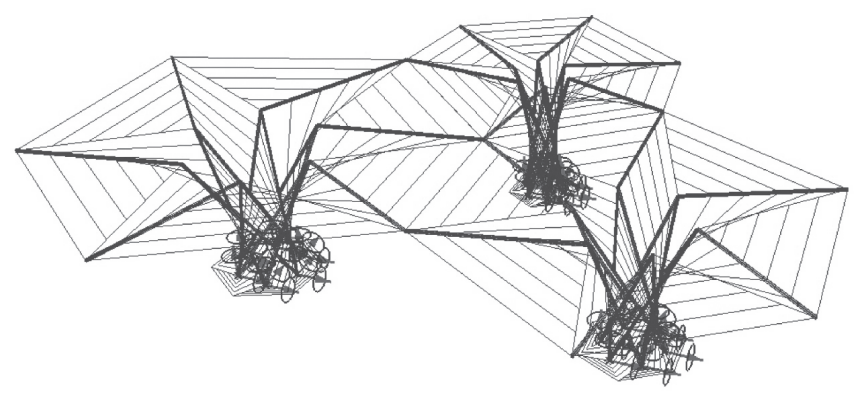

Figure 6: Roof-structure of interconnected HP-surfaces.

\section{Conclusion and Outlook}

Taking into account various aspects of the architectural production at an early stage of the design process enables the architect to avoid complications in a later stage, especially on site. On the other hand a design approach, implementing and connecting all available information in an all-embracing process is very complex. Hence, managing complexity is a for sure one of the major tasks we are facing in our research and practice. Computation allows for an accessible handling of complexity throughout the whole design to build process. Yet, the case studies also showed that the integration of parameters and requirements of functionality, efficiency and fabrication as well as structural/physical performance shift the focus from design to optimization. Especially the further integration and specific conditions for the realization while keeping the focus on the design will be a major aspect for the future work. Against this background the different projects will be continued to make them fit for the fabrication and construction of mock-ups and 1:1 prototypes to proof the particular computational design and construction strategy.

\section{Acknowledgements}

All projects were carried out within the international postgraduate Master program Computational Design and Construction (www.m-cdc.de) at the Hochschule Ostwestfalen-Lippe in Detmold, Germany. Participating students: Manuel Sotomayor, Patric Günther, Kaveh Allhadin and Rodrigo Velasco. Supervisors: Holger Hoffmann, Matthias Michel and Marco Hemmerling.

The three-semester program focuses on computational design strategies and construction technologies in architecture, engineering and design. The curriculum places a particular emphasis on the interfaces between the various disciplines and planning phases. This goes along with an increasing demand for graduates with good qualifications in the interdisciplinary field that can activate information technology for the architectural production process. The aim of the program is to enable students who already hold a first professional degree to acquire in-depth qualified scientific knowledge and approaches of artistic, creative as well as technical and methodical kind.

\section{References}

Jencks, C. (1997), Nonlinear Architecture. New Science = New Architecture?, in: Architectural Design, 129.

Schumacher, P. (2010), The Autopoiesis of Architecture: A New Framework for Architecture, Volume 1, Wiley \& Son.

Tschumi, B. (1995), Responding to the question of complexity, in: Complexity. Art, Architecture, Philosophy, Journal of Philosophy and the Visual Art, Andrew Benjamin (ed.), Nr. 6. London.

Valena, T. a.o. (2011), Structuralism Reloaded. Rule-Based Design in Architecture and Urbanism, Edition Axel Menges.

Venturi, R. (1966), Complexity and Contradiction in Architecture, New York, 228-229.

Engel, H. (1967), Structural Systems, DVA, Stuttgart.

Moussavi, F. (2009), The Function of Form, Actar and Harvard Graduate School of Design.

Voronoi, G. (1908), Nouvelles applications des paramètres continus à la théorie des formes quadratiques, Journal für die Reine und Angewandte Mathematik, 133: 97-178. 